\title{
Endeudamiento y competencia en el mercado: Evidencia de las empresas en México
}

\author{
Manuel Sánchez Valadez*
}

\section{RESUMEN}

Motivado por la importancia que tiene el análisis de las decisiones de las empresas en los diversos mercados donde interactúan, y considerando distintas perspectivas al examinar sus causas o efectos, o ambos, el presente documento plantea que las decisiones financieras de las empresas son influidas por la competencia que éstas enfrentan en los mercados reales. El objetivo principal de este trabajo es aportar evidencia que ayude a cubrir la falta de estudios empíricos acerca de esta interacción. Los resultados hallados aportan indicios acerca de los efectos diferenciados del entorno competitivo en la estructura financiera de las empresas, además corroboran parcialmente la teoría sobre los principales determinantes de la estructura financiera de las empresas, destacando la preferencia de las mismas por el mayor uso de fuentes internas de recursos.

Palabras clave: competencia a la Cournot, competencia a la Bertrand, fuentes de financiamiento, deuda, estrategias competitivas, apalancamiento.

Clasificación JEL: G32, L10.

\begin{abstract}
Motivated by the importance of analyzing decisions of firms in different markets where they interact, and considering different perspectives to examine the causes or effects, or both, this paper argues that corporate financial decisions are influenced by competition they face in real markets. The main objective of this work is to provide evidence to help cover empirical studies lack about this interaction. Results found suggest differential effects of competitive environment on firms financial structure. Moreover, results partially corroborate the theory on the main determinants of corporate financial structure, underscoring preference of companies for increased use of domestic sources of resources.
\end{abstract}

Key words: Cournot competition, Bertrand competition, financing sources, debt, competitive strategies, leverage.

Classification JEL: G32, L10.

* Estudiante del doctorado en Economía Aplicada en la Universidad Autónoma de Barcelona. Agradezco los comentarios de los dictadores anónimos y los comentarios y sugerencias de Francesc Trillas a una versión previa del documento; agradezco también el apoyo financiero del Consejo Nacional de Ciencia y Tecnología (Conacyt). 


\section{INTRODUCCIÓN}

Los datos del Censo Económico, elaborado en 2004 por el Instituto Nacional de Estadística, Geografía e Informática (INEGI), indican que sólo $1.1 \%$ del total de empresas correspondía a empresas medianas y grandes. No obstante el pequeño porcentaje que las empresas medianas y grandes puedan representar respecto del total de las empresas en México, éstas generaron, según el Censo Económico, aproximadamente $77 \%$ del valor de la producción bruta de la economía, y son las mismas que detentan el mayor poder de mercado. Por ello, las acciones emprendidas por este tipo de empresas para afrontar la competencia tienen mayor influencia sobre las relaciones que mantienen en los mercados.

Conocer qué factores determinan la estructura financiera de las empresas y analizar cómo inciden en ella, resulta importante para comprender mejor las reacciones de aquellas ante modificaciones en las condiciones de los mercados y así poder proponer medidas de política que fomenten el desarrollo eficiente de las empresas.

Al respecto, vale recordar que si bien la teoría económica considera la existencia de mercados perfectos, las estructuras de mercado que se desvían de éstos son las que han recibido una mayor atención desde una perspectiva de política económica, puesto que generan efectos negativos en la eficiencia de las actividades económicas. Ante ello, analizar las decisiones de las empresas en los diferentes mercados, desde distintas perspectivas, considerando sus efectos o causas, o ambos, ha sido siempre un aspecto de gran interés para los investigadores.

Por su parte, la forma de financiar la actividad de las empresas ha recibido especial atención desde hace poco más de cincuenta años, cuando fue publicado el documento seminal de Franco Modigliani y Merton Miller (1958), el cual planteó, bajo ciertos supuestos, la inocuidad de las decisiones de financiamiento sobre el valor de las empresas. Años después, a partir de la crítica a lo propuesto por estos autores, se ha tendido un vínculo entre la teoría financiera y la organización industrial.

Por ello, surge la pregunta respecto de la interrelación entre las decisiones financieras de las empresas y las decisiones que éstas toman en los mercados de bienes y servicios -mercados reales-. Es de notar que esta pregunta no es nueva, pues en los veinte años pasados ha tenido lugar una discusión académica en torno de ella, sin embargo, destaca la escasa bibliografía empírica sobre el te- 
ma. ${ }^{1}$ Con lo anterior, el presente documento plantea que las decisiones financieras de las empresas son influidas por la competencia que enfrentan en los mercados reales y sugiere una respuesta a cómo influyen las decisiones de las empresas en los mercados reales sobre las decisiones financieras de éstas. De acuerdo con la teoría, es de esperarse que la influencia dependa del tipo de interacción competitiva que las empresas mantienen en los mercados reales.

Así, el objetivo principal del trabajo es aportar evidencia que ayude a cubrir la falta de estudios empíricos sobre esta interacción. Dado que la evidencia disponible se centra en mercados de economías desarrolladas, principalmente de Estados Unidos, un objetivo adicional del documento es aportar evidencia para el caso de una economía en desarrollo, donde la profundidad e interacción entre los mercados puede ser aún débil. Para ello, se considera una muestra de empresas que cotizan en la Bolsa Mexicana de Valores (BMV).

En cuanto a lo anterior, dos comentarios: Primero, si bien el total de empresas inscritas en la BMV aún es pequeño en relación con otros mercados, ${ }^{2}$ la mayoría corresponde a empresas medianas o grandes, las cuales, por sus volúmenes de producción, sin lugar a dudas desempeñan un papel fundamental dentro de los mercados reales mexicanos. Segundo, cabe mencionar que en el caso de las empresas en México, el autor no conoce, hasta el momento, algún documento con evidencia de la interacción entre las decisiones financieras de las empresas y las decisiones de éstas en los mercados reales.

En este documento se trató de captar el vínculo entre las decisiones financieras y las decisiones de mercado de las empresas a partir de la clasificación de éstas según su entorno competitivo -sustitutos o complementos estratégicosy mediante su reacción ante la incertidumbre en las condiciones de mercado, demanda y costos. Los resultados hallados sugieren que el comportamiento de las empresas en los mercados reales tiene un efecto diferenciado en su estructura financiera.

El resto del documento se divide en cuatro secciones. La sección I expone las bases teóricas de la discusión sobre la estructura financiera de las empresas y el vínculo con los mercados reales. En la sección II se describe la metodología empírica propuesta, incluyendo también la revisión de la bibliografía previa. La

\footnotetext{
${ }^{1}$ Para una revisión de la bibliografía teórica y empírica sobre el tema, véase Franck y Huyghebaert (2004).

${ }^{2}$ En 2008 estaban inscritas en la Bolsa Mexicana de Valores alrededor de 135 empresas, mientras que, por ejemplo, el New York Stock Exchange agrupaba cerca de 2600 empresas.
} 
sección III presenta los resultados obtenidos y, finalmente, se ofrecen las conclusiones del trabajo.

\section{MARCO TEÓRICO}

Uno de los teoremas que fundamentan la teoría de las finanzas corporativas fue postulado en 1958 por Franco Modigliani y Merton Miller, convirtiéndose en un parteaguas respecto a la influencia de las decisiones financieras de las empresas. En específico, éste aborda las consecuencias de la elección de diferentes opciones de financiamiento sobre el valor de las empresas. Casi treinta años después de haberse publicado el documento seminal donde se postuló el conocido teorema Modigliani-Miller (TMM), surgieron trabajos críticos acerca de éste que cuestionaban si realmente no importaba la estructura financiera de las empresas.

Documentos como los de Brander y Lewis (1986), Maksimovic (1988) y Bolton y Schaferstein (1990) se enfocaron en demostrar que relajando los supuestos del TMM, la estructura financiera de las empresas influía relativamente sobre las decisiones que las empresas tomaban en los mercados reales, demostrando que existen vínculos importantes entre los mercados financieros y los mercados reales. Sin embargo, tuvieron que pasar algunos años más para que se publicaran los primeros documentos con evidencia empírica sobre la interrelación entre las decisiones financieras de las empresas y sus decisiones de mercado.

\section{Los postulados del teorema Modigliani-Miller}

La motivación central del documento seminal de Modigliani y Miller (1958) es responder: "¿puede un proyecto incrementar el valor de la empresa, dependiendo de cómo se financie?". Para ello, los autores formularon tres proposiciones basadas en algunos supuestos restrictivos. Al respecto, entre los supuestos se sugiere que las empresas pueden financiar sus proyectos con la emisión de acciones o de bonos. Además, se supone que las empresas pueden dividirse en clases, $k$, de rendimientos equivalentes, debido a que el rendimiento de las acciones emitidas por una empresa de cualquier clase es proporcional al rendimiento de las acciones emitidas por otra empresa de la misma clase. ${ }^{3}$

\footnotetext{
${ }^{3}$ Este último supuesto implica que la rentabilidad de las acciones emitidas por una empresa se determina por un factor de escala o tasa de capitalización, correspondiente a su clase de riesgo $\left(\rho_{k}\right)$. Lo anterior permite clasificar a las empresas en grupos, donde las acciones de diferentes empresas son homogéneas y perfectas sustitutas entre ellas.
} 
La primera de las proposiciones, la parte más conocida del TMM, menciona que "el valor de mercado de una empresa es independiente de su estructura de capital y se determina por la capitalización de sus rendimientos esperados a la tasa $\rho_{k}$ de acuerdo con la clase $k$ a la cual pertenece". Esto significa que el costo promedio del capital de una empresa es completamente independiente de su estructura de capital y es igual a la tasa de capitalización de sus acciones, según la clase a la que pertenezca.

La segunda proposición del TMM menciona que "la tasa de rendimiento esperado, $i$, sobre las acciones de una firma $j$ perteneciente a una clase $k$, es una función lineal del apalancamiento financiero de tal firma". Mientras que la tercera proposición del TMM enuncia que "si una empresa de la clase $k$ persigue la obtención de los máximos beneficios para sus accionistas en el momento de tomar las decisiones, ésta explotará una oportunidad de inversión si y sólo si la tasa de rendimiento de la inversión, denotada por $\rho^{*}$, es mayor o igual a $\rho k^{\prime}$. Así, las decisiones de inversión de una empresa se tomarán con base en $\rho k$ y en ningún caso serán afectadas por el tipo de financiamiento utilizado.

De estas tres proposiciones se desprende con claridad vislumbrar una de las conclusiones del TMM, según la cual las decisiones financieras de las empresas no afectan el valor de la misma. Por tanto, no importa cómo se financian las empresas, si a partir de deuda o con la emisión de acciones, su valor no será afectado.

En otras palabras, tal como lo menciona Tirole (2006), las decisiones financieras influyen en "cómo se reparte el pastel" mas no en el "tamaño del pastel". Es decir, la única influencia que pueden tener las decisiones financieras de las empresas es sobre cómo se distribuye la forma en que financia sus proyectos sin que ello afecte el valor de la empresa.

\section{Los factores determinantes de la estructura financiera}

Teniendo presentes las conclusiones del TMM algunos economistas se cuestionaron sobre la importancia de la estructura financiera de las empresas. Ante ello, surgen dos preguntas: ¿qué factores determinan la estructura de capital de las empresas?, ¿existe una estructura de capital óptima para las empresas? Con el objetivo de dar respuesta a estas preguntas se relajaron algunos supuestos del TMM, dando lugar con ello a vertientes diferentes que intentan responder estas cuestiones.

Dada la diversidad de aproximaciones al tema, como Myers (2001) lo menciona, no hay una teoría universal acerca de la estructura financiera de la 
empresa ni razón para esperarla, puesto que cada teoría está "condicionada" por los supuestos adoptados. No obstante, a fin de acotar las aproximaciones, según Harris y Raviv (1991), éstas pueden agruparse en cinco diferentes enfoques.

El primero es la teoría del equilibrio estático, la cual propone que los factores determinantes de la estructura de capital están relacionados con un trade-off entre las posibles ventajas fiscales de mantener cierto nivel de apalancamiento y los costos de insolvencia que esto podría provocar.

Un segundo enfoque lo constituyen documentos que consideran la existencia del problema agente-principal. Con éste, la estructura financiera de la empresa podría reflejar la no coincidencia de incentivos entre administradores y accionistas, o dueños, de la empresa, o entre accionistas y acreedores y, por tanto, la estructura estaría condicionada por los incentivos de algunos de los agentes. ${ }^{4}$

El tercero es el referente a las posibles asimetrías de información, entre agentes internos y externos de la empresa. ${ }^{5}$ Es decir, los agentes internos conocerán el "verdadero estado" de la empresa respecto del riesgo que ésta enfrenta y los flujos que puede generar, mientras que los agentes externos pedirán el pago de una prima de riesgo como compensación del desconocimiento de esta información. ${ }^{6}$

Un cuarto enfoque es el basado en el control corporativo de la empresa. Según éste, la estructura financiera de la empresa puede verse afectada por la respuesta a una oferta pública de adquisición (OPA), con lo que se busque no perder el control de la empresa y se prefieran mayores niveles de endeudamiento.

El quinto enfoque se basa en las posibles estrategias de mercado utilizadas por las empresas para enfrentar la competencia.

\footnotetext{
${ }^{4}$ El primer conflicto de incentivos mencionado tiene que ver con la conducta maximizadora de los administradores, mientras que el segundo está relacionado con una diferencia de responsabilidades de cada uno de los agentes ante pérdidas de la empresa. Este último es el conflicto entre accionistas y tenedores de deuda, en el cual la contratación de deuda incentiva a los accionistas a tener un nivel subóptimo de inversión.

${ }^{5}$ Los agentes internos afectan las actividades de la empresa (empleados propietarios, etcétera), mientras que los agentes externos son afectados por las actividades de la empresa (acreedores, proveedores, gobierno, etcétera). En este caso, la asimetría de información influye en la elección del instrumento de financiación y, por tanto, en la estructura de capital al intentar minimizar el pago de la prima de riesgo.

${ }^{6}$ Una de las teorías contenidas en este enfoque es la teoría de la jerarquía en la toma de decisiones o pecking order theory, la cual postula que la empresa pedirá prestado, antes que emitir capital, cuando los flujos de efectivo internos no sean suficientes para financiar proyectos de inversión, Myers (2001).
} 


\section{Las decisiones financieras y las decisiones de mercado}

Profundizando en este último enfoque, que considera las estrategias de mercado para hacer frente a la competencia, se tiene que la interacción entre la estructura de capital y las decisiones en los mercados reales puede ser de dos tipos. Por una parte, está la que influye sobre la estrategia de mercado de la empresa y, por otro, la que afecta la elección del producto (Harris y Raviv, 1991).

Considerando los efectos provocados por cambios en la estrategia de la empresa, la interacción entre los mercados reales y la estructura financiera de las empresas, se debe a que los instrumentos financieros alteran la propiedad de los flujos de efectivo y, con ello, la capacidad de inversión de una empresa. Estos cambios afectan las creencias y expectativas que un competidor o competidores se crean sobre las acciones potenciales que una empresa pueda llevar a cabo (Phillips, 1995). ${ }^{7}$

De acuerdo con Brander y Lewis (1986), la existencia de obligaciones de la empresa con acreedores y deudores podría generar un desequilibrio financiero (financial distress), que resultaría en cambios en las estrategias de mercado de la empresa, los cuales son percibidos por sus rivales. ${ }^{8}$

Al revisar la bibliografía sobre la interacción de las decisiones de competencia de la empresa y las decisiones financieras, se pueden identificar claramente tres posiciones teóricas diferentes (Phillips, 1995). La primera aborda el efecto estratégico de la quiebra, también conocida como efecto de "cartera profunda" (deep purse); la segunda revisa la relación entre la estructura de capital y el efecto de la inversión, en tanto la tercera vincula la producción y el efecto de responsabilidad limitada.

Según la primera de las posiciones teóricas mencionadas, deep purse, el vínculo entre la estructura financiera y la competencia se basa en que una empresa rival podría aumentar su producción con el fin de incrementar la probabilidad de quiebra de empresas altamente endeudadas. ${ }^{9}$ En este contexto, el término de

\footnotetext{
${ }^{7}$ Con la compra-venta de títulos, la propiedad de una empresa se ve alterada, con lo que sus incentivos pueden variar y, con ello, la estrategia de competencia en el mercado. Por ello, los rivales de la empresa modificarán sus estrategias para enfrentar la nueva estrategia competitiva.

${ }^{8}$ La incorporación de las interacciones estratégicas de las empresas en los mercados de bienes ante cambios financieros, hace que el análisis económico se aproxime más al funcionamiento real de los mercados.

${ }^{9}$ Este argumento sólo tiene sentido al existir restricciones de financiamiento, de otra manera, las empresas pueden recurrir al mercado cuando se enfrentan a comportamientos predatorios de sus rivales.
} 
“cartera profunda" implica para la empresa un acceso casi ilimitado a recursos financieros, permitiendo soportar pérdidas y mantener solvencia aun ante choques negativos.

La segunda posición teórica muestra que con altos niveles de deuda, los costos de expansión de la producción son mayores, dado que cada vez es menor la posibilidad de utilizar las reservas por ganancias acumuladas y los flujos de efectivo residuales. ${ }^{10} \mathrm{El}$ incremento en los costos de la expansión de la producción de una empresa podría ser utilizado por sus rivales, quienes buscarán aumentar su propia producción con el fin de disminuir los beneficios de la empresa altamente endeudada, provocando su salida del mercado ante la insuficiencia de recursos para incrementar la producción. ${ }^{11}$

\section{a) Primeros modelos}

A partir de la tercera posición teórica, la cual vincula las decisiones de producción y el efecto de responsabilidad limitada, se desprende uno de los modelos seminales en el tema (Brander y Lewis, 1986). Estos autores suponen una interacción de las empresas en un mercado de tipo Cournot, centrando su modelo en la capacidad que pueda tener la estructura de capital para alterar los beneficios de los accionistas. ${ }^{12}$ En este modelo se asume que una vez determinada la estructura de capital de las empresas, éstas eligen su nivel de producción. Para mostrar las diferentes reacciones que la empresa puede tener, se supone que, una vez de-terminados el nivel de producción y la estructura de capital, los beneficios marginales de la empresa sufren un choque aleatorio, que puede ser positivo, incrementando los beneficios de la empresa, o negativo, reduciendo los beneficios marginales de la empresa.

Con el fin de maximizar los beneficios de las empresas, un incremento en la deuda lleva a que éstas elijan estrategias que aumenten los beneficios en el caso de realizaciones positivas del choque y minimicen los beneficios ante realizaciones negativas de éste. Asumiendo que los accionistas tienen responsabili-

\footnotetext{
${ }^{10}$ Las reservas por ganancias acumuladas y los flujos de efectivo residuales representan para la empresa fuentes más baratas de financiamiento, en comparación con recursos externos.

${ }^{11}$ Esta explicación considera la existencia de altos costos de la financiación externa, lo que parcialmente podría ser resultado de problemas de información asimétrica.

${ }^{12}$ Este modelo no distingue entre los administradores y los accionistas de la empresa, por lo que en principio se elimina un posible problema agente-principal entre éstos.
} 
dad limitada y son "reclamantes residuales" de los beneficios de la empresa, Brander y Lewis (1986) encuentran que con incrementos de deuda las empresas siguen una estrategia agresiva, buscando la maximización de beneficios de los accionistas. $^{13}$

Por su parte, Showalter (1995), para probar los efectos estratégicos de la deuda, asume que las empresas compiten en un mercado del tipo Bertrand. Sugiriendo que, ante este tipo de entorno competitivo, los niveles de deuda de las empresas podrían ser instrumentos estratégicos dependiendo del tipo de incertidumbre que exista en el mercado. Es decir, la respuesta estratégica será diferente ante incertidumbre de la demanda o de costos, por lo que los niveles de deuda de una empresa tendrán efectos distintos en el mercado. De esta manera, ante incertidumbre de los costos las empresas no utilizarán la deuda como una estrategia de mercado, mientras que con incertidumbre de la demanda, los niveles de deuda serán positivos. ${ }^{14}$

Las conclusiones de los modelos mencionados contrastan entre sí. Brander y Lewis (1986) sugieren que los niveles de deuda de las empresas serán positivos, no importando el tipo de incertidumbre del mercado, dando como resultado estrategias de mercado más agresivas. Mientras que Showalter (1995) sugiere que el tipo de estrategia competitiva dependerá del tipo de incertidumbre en el mercado.

Con lo anterior, se tiene que relajando los supuestos del TMM, con el fin de definir los factores determinantes de la estructura financiera de las empresas, algunos autores profundizaron en el vínculo entre las decisiones competitivas de las empresas en los mercados reales y las decisiones financieras, construyendo así un nexo entre la teoría financiera y la organización industrial.

\footnotetext{
${ }^{13}$ Maksimovic (1988) amplía el modelo de Brander y Lewis (1986), incorporando más periodos de interacción en el análisis y considerando la elasticidad de la demanda, el número de competidores en el mercado y la tasa de descuento, concluyendo que un aumento de la deuda dificulta mantener ingresos que permitan la colusión entre empresas.

${ }^{14}$ Showalter (1995) supone que la competencia entre las empresas puede definirse en términos de lo que Bulow et al. (1985) denominan sustitutos estratégicos y complementos estratégicos. Sundaram et al. (1996) mencionan que la competencia en sustitutos estratégicos implica un comportamiento tal que los competidores de una empresa dan cabida a los cambios en la estrategia de la empresa, por lo que se comportan "más amables". Mientras en la competencia en complementos estratégicos, los competidores de la empresa "van igualando" los cambios en la estrategia de la empresa; el resultado es un incremento de la competencia.
} 


\section{b) Un modelo con interacción competitiva}

Evgeny Lyandres (2006) propuso examinar el vínculo entre el grado de interacción competitiva entre las empresas, su apalancamiento óptimo y la agresividad de sus estrategias operativas. Para ello, retoma las conclusiones de Brander y Lewis (1986) y Showalter (1995), aclarando que ninguno de los documentos analiza la relación entre el grado de interacción competitiva entre rivales de mercado y la estructura de capital óptima.

El modelo de Lyandres (2006) considera dos periodos. En el primero, las empresas coinciden en el mercado y de manera simultánea eligen sus estructuras financieras, es decir, los accionistas eligen su nivel óptimo de deuda con el objetivo de maximizar el valor de la empresa. La decisión sobre el nivel de deuda se realiza tomando en consideración tanto su propia estrategia a seguir como la de su rival en el mercado. ${ }^{15}$ En el segundo periodo, los accionistas de cada empresa eligen sus estrategias en el mercado de bienes antes de enfrentarse a un choque en el flujo de caja. La concreción del choque, junto con las estrategias de mercado elegidas por la empresa y su rival, determinan la solvencia o insolvencia de la empresa. ${ }^{16}$

El efecto estratégico de la deuda resulta del hecho de que una vez determinados los niveles de la deuda, al elegir la estrategia de mercado los accionistas sólo consideran choques que lleven a la empresa a estados de solvencia. Es decir, no se considera la posibilidad de choques que deriven en una situación de insolvencia de la empresa (default), convirtiendo de esta manera la deuda en un mecanismo de compromiso para elegir estrategias competitivas más agresivas en el segundo periodo.

Lyandres (2006) demuestra que existe un único equilibrio estable en la elección de las estrategias de las empresas, en donde con sustitutos estratégicos, la agresividad de la estrategia competitiva disminuirá en el valor nominal de la deuda del rival. Mientras que para los complementos estratégicos el efecto es exactamente el contrario.

Es posible apreciar que la deuda tiene el efecto estratégico de inducir en

\footnotetext{
${ }^{15}$ Dado que Lyandres (2006) asume que los administradores actúan de acuerdo con los intereses de los accionistas, se elimina la existencia de un problema agente-principal.

${ }^{16}$ La empresa es solvente si el choque sobrepasa el umbral del valor de la empresa, por lo que puede hacer frente a sus obligaciones de deuda y distribuir el flujo residual de caja entre los accionistas. Mientras que si el choque no alcanza dicho umbral, no se genera un flujo de caja suficiente, la deuda no se paga y la empresa cae en default.
} 
el rival de una empresa la elección de una estrategia menos agresiva (en el caso de que compitan en sustitutos estratégicos) o bien provocar que el rival asuma una estrategia de producción más agresiva (en el caso de competencia en complementos estratégicos), por lo que el beneficio estratégico de la deuda está en el cambio de estrategia del rival.

Lyandres (2006) también propone la existencia de un equilibrio único y estable del nivel de deuda de las empresas, el cual establece la relación de equilibrio entre las decisiones financieras de las empresas y el grado de interacción competitiva de las industrias a las que pertenecen las empresas. ${ }^{17}$ Una de las conclusiones del documento de Lyandres es que la deuda tiene un beneficio estratégico, que se incrementa conforme aumenta el grado de interacción competitiva. Con ello, el apalancamiento de la empresa crece a medida que aumenta el grado de interacción competitiva.

\section{METOdologíA EMPÍRICA}

La sección anterior abordó el teorema Modigliani-Miller y la discusión surgida del relajamiento de los supuestos que lo apuntalan dio como resultado la aparición de diferentes enfoques sobre los factores que inciden en la estructura financiera de las empresas. Dentro de estos enfoques, está la inclusión de las estrategias competitivas en el mercado real como factor determinante. Al respecto, si bien hay una relativa diversidad de trabajos teóricos que consideran las estrategias de mercado de las empresas como un factor determinante más de su estructura financiera, pocos son los documentos que abordan la evidencia empírica sobre el tema (Franck y Huyghebaert, 2004).

\section{Trabajos empíricos previos}

Uno de los primeros documentos que aporta evidencia empírica respecto del vínculo entre decisiones financieras de las empresas y la competencia en el mer-

\footnotetext{
${ }^{17}$ Lyandres (2006) demuestra que este equilibrio se determina en parte por el valor de un parámetro de interacción entre las empresas, que parte de la definición del umbral de default de las empresas expresado como $T_{1}=c_{1}+r c_{2}$, en donde $c_{1}$ y $c_{2}$ corresponden a las estrategias competitivas de las empresas y $r$ es el parámetro de interacción, el cual presenta el nivel de influencia de las decisiones de la empresa sobre el valor de sus competidores. A este parámetro de interacción se le permite un intervalo de variación entre -1 y 1 . Con ello, si $r>0$, las empresas compiten en sustitutos estratégicos, mientras que si $r<0$, las empresas compiten entonces en complementos estratégicos.
} 
cado es el de Chevalier (1995). En este trabajo, utilizando datos de supermercados en Estados Unidos, se analizan los efectos de la recapitalización sobre las estrategias de mercado de las empresas. Las conclusiones de la autora sugieren que el incremento en el nivel de apalancamiento de las empresas hacia finales de los años ochenta resultó en una competencia menos agresiva, "más suave", en la rama de los supermercados.

Bajo la misma idea de analizar los efectos de la recapitalización de las empresas sobre las decisiones de cambios en precios y cantidades, Phillips (1995) estudia cuatro industrias concentradas en Estados Unidos que experimentaron cambios relativamente considerables en los niveles de apalancamiento de las empresas que las conformaban. Los resultados hallados sugieren que la estructura de capital afecta las decisiones de producción de la empresa y las de sus rivales, puesto que ésta puede actuar con un compromiso de no efectuar inversiones y, por tanto, mantener una estrategia competitiva menos agresiva.

Por su parte, Zingales (1998) estudia los efectos de las imperfecciones del mercado de capital sobre la selección de las empresas más eficientes por parte del propio mercado y encuentra que las empresas con mayor apalancamiento tienen menor probabilidad de mantenerse en el mercado ante un proceso desregulatorio. En tanto, Dean Showalter (1999) aporta evidencia acerca del uso de la deuda como estrategia de mercado. Este autor estudia las empresas en la industria manufacturera y su hallazgo consiste en que éstas incrementan su deuda conforme aumenta la incertidumbre en la demanda, pero se reduce cuando crece la incertidumbre en los costos, corroborando así lo propuesto en su documento de 1995.

Mackay y Phillips (2005) analizan también el efecto de pertenencia de una empresa a una determinada industria sobre las decisiones de las empresas en los mercados reales y financiero. Estos autores encuentran que la estructura financiera de una empresa depende también de la posición que ésta tenga al interior de la industria. En el trabajo de Lyandres (2006) se incluye el grado de interacción competitiva de las empresas como determinante de su estructura financiera, concluyendo que el grado de interacción competitiva es un determinante importante de su estructura financiera.

Jong et al. (2007) estudian la influencia de la incertidumbre en la demanda y en los costos sobre las decisiones financieras. Los resultados de estos autores sugieren que la incertidumbre en la demanda está positivamente relacionada con la estructura financiera de las empresas, sin importar el tipo de entorno competitivo -Bertrand o Cournot-, mientras que la incertidumbre en costos sólo afecta la estructura financiera de las empresas en un entorno competitivo a la Cournot. 
Por otra parte, en la búsqueda de bibliografía sobre el tema se hizo especial hincapié en encontrar documentos con evidencia empírica para el caso de las empresas en México. Al respecto, es de mencionar el trabajo de Rodríguez Matilla (2002), que define los principales factores determinantes de la estructura de capital de las empresas en México; sin embargo, éste no aborda la interrelación entre las decisiones financieras y las decisiones de mercado de la empresa.

\section{Datos, metodologías y variables}

\section{a) Datos}

Los datos disponibles corresponden a la información financiera de 205 empresas no financieras listadas en la BMV entre el tercer trimestre de 1998 y el segundo trimestre de 2008. Al respecto, cabe señalar que si bien el número de las empresas inscritas en la BMV entre 1998 y 2007 ha disminuido (pasando de 195 a 135 empresas), su valor de capitalización se ha incrementado, al pasar de alrededor de $24 \%$ del PIB en 1998 a poco más de $44 \%$ en 2007 (véase gráfica 1). ${ }^{18}$

Gráfica 1. Valor de capitalización de las acciones de empresas que cotizan en la BMV (Porcentaje del PIB)

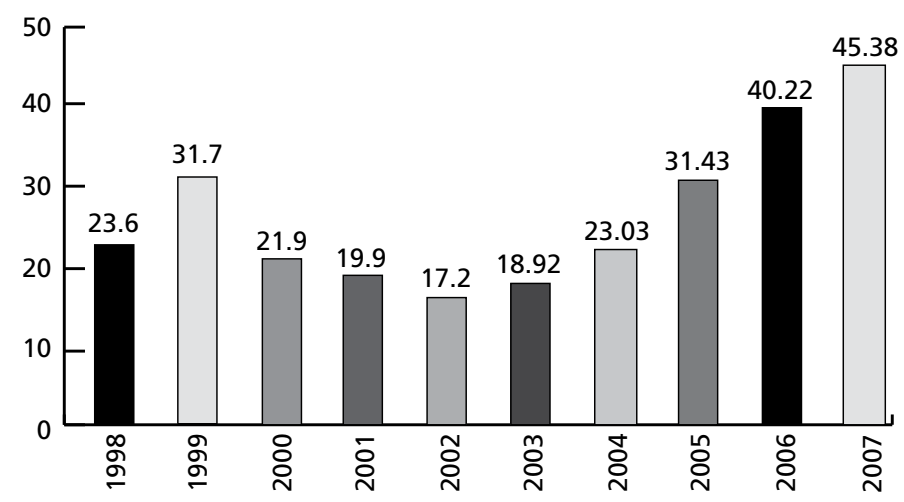

Fuente: Elaboración a partir del Segundo Informe de Gobierno 2008 y del Informe Anual de la Bolsa Mexicana de Valores 2007.

${ }^{18}$ El valor de capitalización es el valor de las acciones cotizadas en un momento determinado. Desafortunadamente, no se cuenta con la información de los precios de las 205 empresas comprendidas en la muestra, por lo que no fue posible calcular su valor de capitalización específico. 
Por otra parte, si bien los datos sólo corresponden a empresas inscritas en la BMV y en ese sentido no representan la totalidad de las empresas en la economía mexicana, es necesario aclarar algunos aspectos relacionados con el tipo y tamaño de las empresas consideradas. Primero, no se incluyen empresas financieras, principalmente bancos, pues sus niveles de apalancamiento, como indicador indirecto de su estructura financiera, no tienen relación alguna con los niveles de apalancamiento de las empresas no financieras debido tanto a la propia naturaleza de sus actividades como a la regulación a que están sujetas en cuanto al nivel de capitalización requerido. Segundo, las empresas inscritas en la BMV, de acuerdo con la información disponible, corresponden a empresas medianas y principalmente grandes. Si bien este tipo representa un pequeño porcentaje del total de las empresas en la economía mexicana, aportan un alto porcentaje del valor de la producción bruta de la economía mexicana. Tercero, las empresas no comprendidas en la muestra, por sus propias características, poco pueden incidir sobre el comportamiento del mercado. ${ }^{19} \mathrm{Al}$ respecto, si bien el número de las micro y pequeñas empresas puede representar un porcentaje muy alto respecto del total de empresas de la economía mexicana, las decisiones de cada una de éstas en poco podrían desviar las conductas del mercado, caso contrario de algunas empresas grandes consideradas en la muestra.

Con los datos disponibles, se construyó un panel, buscando tener la máxima información posible de diversos mercados. Este panel se formó con 91 empresas entre el cuarto trimestre de 2001 y el primero del 2007. De acuerdo con los sectores propuestos por la BMV, en el panel se incluyen 35 empresas del sector de la transformación, 13 de la construcción, 17 del sector comercio, 10 de comunicaciones y transportes, 8 del sector servicios y 8 más de otros sectores.

Los datos de las 91 empresas del panel muestran que en 2001 éstas representaban $49 \%$ del total de las empresas inscritas en la BMV y el valor de sus ventas significaba $21 \%$ del PIB, mientras que en 2006 estos porcentajes ascendieron a $65 \%$ del total de empresas aparecidas en la BMV y a $28 \%$ del PIB (véase gráfica $2 \mathrm{a}$ y gráfica $2 \mathrm{~b}$ respectivamente).$^{20}$ Por su parte, el apalancamiento promedio de la muestra fue de alrededor de $49 \%$ de los activos totales (gráfica 2c).

${ }^{19}$ Micro y pequeñas empresas, que por su tamaño, volumen de inversiones y estructura, no cumplen los requisitos para poder inscribirse y cotizar en la Bolsa Mexicana de Valores.

${ }^{20}$ Este porcentaje es respecto al PIB, sin incluir agricultura, silvicultura y pesca, electricidad, gas y agua y servicios financieros y seguros, sectores de los cuales no se tienen empresas en la muestra. Además, por el desglose de la información disponible no se corrigió por el PIB petrolero. 


\section{Gráfica 2. Características del panel}

a) Número de empresas

Porcentaje del número de empresas inscritas en la BMV b) Ventas netas

Porcentaje del PIB* c) Apalancamiento promedio

Pasivos totales como porcentaje de los activos totales
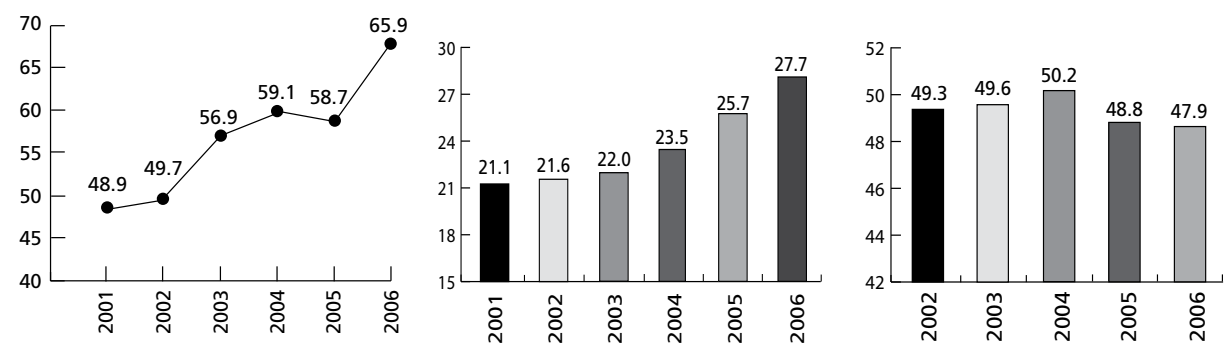

* No incluye el PIB de agricultura, silvicultura y pesca, electricidad, gas y agua, servicios financieros y seguros. Por el desglose de la información disponible no se corrigió por el PIB petrolero.

Fuente: Elaboración propia a partir de datos de la BMV y del INEGI.

Debido al desglose de la información disponible no se corrigió por el PIB petrolero. Dado que se pretende analizar la influencia del entorno competitivo sobre las decisiones financieras de las empresas, es necesario identificar los mercados en los cuales las empresas operan. Para ello, se utilizó la clasificación industrial propuesta por Naciones Unidas: Standard Industrial Classification (SIC, por sus siglas en inglés).

En este contexto, una relación de competencia es entendida como el posible contacto en el mercado entre dos o más empresas clasificadas bajo el mismo grupo SIC a dos dígitos. ${ }^{21}$ Con las empresas incluidas en el panel se pudieron formar 18 relaciones de competencia (cuadro 1). Cabe aclarar que las 16 empresas de la muestra que forman el grupo 20 de la clasificación SIC no se incluyeron en el análisis, debido a la poca significación de las relaciones de competencia entre ellas. ${ }^{22}$

${ }^{21}$ Cabe señalar que, debido al tamaño de la muestra disponible, si se considera el criterio del SIC a cuatro dígitos para clasificar a las empresas, las relaciones de competencia encontradas disminuyen de manera importante. No obstante el criterio adoptado, la muestra contiene algunas relaciones de competencia muy claras, como la industria del cemento o de la telefonía celular.

${ }^{22} \mathrm{El}$ grupo 20 de la SIC corresponde a manufacturas, alimentos y productos similares. En este grupo, y de acuerdo con el criterio de clasificación, se podrían estar considerando relaciones de competencia entre empresas que no tienen contacto alguno en el mercado. Sin embargo, en otros casos dentro del panel se consideran mercados casi completos, por ejemplo el grupo que incluye a la industria del cemento, de la cual se tiene $70 \%$ del total de empresas que conforman la industria en México. 
24 ECONOMÍA: TEORÍA Y PRÁCTICA • Nueva Época, número 31, julio-diciembre 2009

\section{Cuadro 1. Relaciones de competencia}

Grupos SIC considerados

\begin{tabular}{|l|l|l|}
\hline Metal minería & Químicos y productos relacionados & $\begin{array}{l}\text { Tiendas de mercancías } \\
\text { generales }\end{array}$ \\
\hline Construcción general & $\begin{array}{l}\text { Piedra, vidrio y productos de } \\
\text { concreto }\end{array}$ & Tiendas de alimentos \\
\hline $\begin{array}{l}\text { Construcción de obras } \\
\text { de gran tamaño }\end{array}$ & $\begin{array}{l}\text { Impresión, publicación y } \\
\text { productos relacionados }\end{array}$ & $\begin{array}{l}\text { Restaurantes y lugares de } \\
\text { venta de alimentos y bebidas }\end{array}$ \\
\hline Productos textiles & Equipo de transporte & Venta de misceláneos \\
\hline Papel y productos relacionados & Comunicaciones (televisión) & Holdings \\
\hline Industrial metálicas primarias & Comunicaciones (telefonía celular) & Hoteles \\
\hline
\end{tabular}

\section{b) Metodologías}

La teoría sugiere que la influencia de las decisiones de mercado sobre las decisiones financieras dependerá del entorno competitivo que enfrentan las empresas (Showalter, 1995; Lyandres, 2006, y Jong et al., 2007). Para aislar los efectos se requiere determinar el tipo de entorno competitivo enfrentado. Así, para clasificar a las empresas de acuerdo con su entorno competitivo es necesario contar con un indicador indirecto del grado de interacción competitiva entre ellas. De esta manera se adoptó la medida de competencia estratégica (CSM) sugerida por Sundaram et al. (1996) para tal fin.

La CSM propuesta por Sundaram et al. (1996) pretende medir hasta qué punto son mutuamente afectadas las estrategias de mercado de una empresa y la de sus competidores. Este indicador se define como el coeficiente de correlación entre el cociente del cambio en los ingresos de una empresa y el cambio de sus ventas, y el cambio de las ventas de sus competidores, en donde las variaciones en las ventas y los ingresos de las empresas intentan reflejar cambios en las estrategias de mercado de las empresas. Formalmente, la CSM es:

$$
C S M_{x}=\operatorname{corr}\left[\frac{\Delta \pi_{x}}{\Delta S_{x}} ; \Delta S_{y}\right]
$$

donde $\Delta \pi_{x}$ y $\Delta S_{x}$ son el cambio en ingresos y ventas de la empresa " $\mathrm{x}$ ", mientras que $\Delta S_{y}$ es el cambio de ventas de sus competidores.

Puesto que la CSM es un coeficiente de correlación, ésta tendrá un rango de variación definido entre -1 y 1 . De esta manera, si el valor de la CSM es menor 
a -0.05 las empresas estarán compitiendo en un entorno de sustitutos estratégicos (Sundaram et al., 1996; Lyandres, 2006, y Jong et al., 2007).

En el caso de sustitutos estratégicos, que correspondería a un entorno de tipo Cournot, ante cambios en la estrategia de mercado de sus rivales, la empresa reaccionará dando cabida a dichos cambios, con lo que su respuesta será "más amable", es decir, se esperaría que los cambios en sus estrategias de mercado fueran menos agresivos; con ello, la CSM debería de presentar valores negativos (cuadro 2). Cabe recordar que la competencia a la Cournot se da por la determinación de las cantidades en el mercado. Se trata de industrias en donde es difícil ajustar su capacidad de producción.

Si la CSM es mayor a 0.05, el entorno competitivo es complemento estratégico, el cual corresponde a la competencia a la Bertrand. Así, las respuestas de las empresas ante cambios en las estrategias de mercado de sus rivales, serán estrategias de mercado más agresivas, por lo que la CSM deberá ser positiva (cuadro 2). Como se sabe, la competencia a la Bertrand es por la determinación del precio en el mercado; a las empresas que compiten en este entorno les resulta más fácil la modificación de la capacidad de producción.

Cuadro 2. Criterios de la CSM

\begin{tabular}{|c|l|}
\hline CSM $<-0.05$ & Sustitutos estratégicos \\
\hline$-0.05<$ CSM $<0.05$ & Sin clasificar \\
\hline CSM $>0.05$ & Complementos estratégicos \\
\hline
\end{tabular}

Con las empresas clasificadas de acuerdo con el entorno competitivo enfrentado, para el análisis econométrico en este documento se utilizó un modelo de datos de panel. Uno de los objetivos de la metodología de los modelos de datos de panel es capturar la relativa heterogeneidad no observable con otros métodos, tanto entre las unidades de estudio como también en el tiempo.

Además, por construcción, este tipo de modelos permite disponer de un mayor número de observaciones, incrementando con ello los grados de libertad y reduciendo la colinealidad entre las variables explicativas y, en última instancia, mejorando la eficiencia de las estimaciones. Por otra parte, al incorporarse en el análisis la heterogeneidad entre las unidades, los estimadores obtenidos son insesgados. Así, la propia incorporación de la heterogeneidad de las unidades hace que los modelos de datos de panel, en comparación con los modelos de corte transversal, sean más flexibles para modelar las diferencias de comportamiento entre los individuos (Greene, 2000). 
Existen dos tipos de modelos de datos de panel: aquellos que suponen que los impactos de las variables explicativas y de las características propias de cada unidad son diferentes (modelo de efectos aleatorios) y los que asumen que las variables explicativas afectan por igual a las unidades de corte transversal y que éstas se diferencian por características propias de cada una de ellas (modelo de efectos fijos); este último tipo de modelo fue el que se estimó en este documento. ${ }^{23}$

\section{c) Variables}

De acuerdo con la evidencia mostrada en documentos anteriores el apalancamiento es un indicador indirecto de las decisiones financieras que reflejan la estructura financiera de la empresa. Esta variable se definió como el cociente entre pasivos totales y activos totales (LEVB). ${ }^{24} \mathrm{Al}$ considerar los pasivos totales, este indicador indirecto capta las decisiones financieras de las empresas evitando posibles distorsiones en los niveles de endeudamiento causadas por el pago del vencimiento de deuda de corto plazo.

En el caso de las variables independientes, las estimaciones consideran indicadores indirectos de los principales factores determinantes de la estructura financiera de las empresas. Las definiciones sugeridas de las variables son:

Liquidez. Definida como el cociente entre activos circulantes y pasivos circulantes. La teoría predice que esta variable deberá tener un efecto negativo en el nivel de endeudamiento de las empresas. Es decir, se esperaría que mientras las empresas mantengan una posición más líquida, buscarán contratar o emitir menos deuda, pues los recursos líquidos representarían una fuente de financiamiento más barata para las empresas.

Rentabilidad. Esta variable se estimó utilizando el cociente entre el resultado de operación y los activos totales. El indicador indirecto de esta variable intenta

\footnotetext{
${ }^{23}$ En el caso del modelo de efectos fijos, la heterogeneidad no observable se incorpora en la ordenada al origen del modelo, mientras que en el modelo de efectos aleatorios, dicha heterogeneidad se recoge en el término de error. Por otra parte, como criterio estadístico de discriminación entre el modelo de efectos fijos y efectos aleatorios, se aplicó el test de Hausman.

${ }^{24}$ Los activos totales se componen de activo circulante, activo de largo plazo, inmuebles, plantas y equipo, activo diferido y otros activos. Mientras que los pasivos totales contienen el pasivo circulante, pasivo de largo plazo, créditos diferidos y otros pasivos.
} 
captar los efectos provocados por los dividendos de la empresa sobre la estructura financiera de la misma. La teoría predice que esta variable debería tener un efecto negativo sobre la estructura financiera de la empresa, puesto que con una mayor rentabilidad las empresas obtendrían más flujos internos, que son recursos más baratos $\mathrm{y}$, por tanto, podrían preferir no contraer deuda.

Tamaño de la empresa. El indicador indirecto de esta variable se construyó con el logaritmo de las ventas netas. El efecto de esta variable sobre la estructura financiera predicho por la teoría es ambiguo, pues por un lado hay enfoques teóricos que sugieren que el efecto del tamaño de la empresa sería negativo dado que las empresas grandes podrían generar mayores flujos para financiar sus proyectos productivos, en tanto otros enfoques predicen un efecto positivo, pues empresas de mayor tamaño tendrían mayor capacidad de endeudamiento y menores costos de bancarrota.

Riesgo. Como indicador indirecto del riesgo de la empresa se utilizó la desviación estándar del indicador indirecto de rentabilidad. Los diferentes enfoques teóricos predicen un efecto negativo de esta variable sobre la estructura financiera de las empresas, pues ante mayor volatilidad de los beneficios de una empresa los costos enfrentados por un desequilibrio financiero serían más altos, generando menos incentivos para incrementar sus niveles de deuda.

Impuestos. Siguiendo lo sugerido por Jorgensen y Terra (2003), se tomó como indicador indirecto el cociente entre la diferencia del resultado antes de impuestos y el resultado neto. No obstante que algunos enfoques teóricos predicen que esta variable tiene un efecto positivo en la estructura financiera de las empresas, otros sugieren que el efecto es indefinido. En general, esta variable trataría de captar las ventajas impositivas de mantener un cierto nivel de deuda.

Colateral o medida de activos fijos. Esta variable se definió como el cociente entre activos fijos y activos totales. Los diferentes enfoques teóricos sugieren que esta variable tiene un efecto positivo en la estructura financiera de las empresas debido a que las empresas con más activos colaterales tienen mayor capacidad de endeudamiento. Una forma de captar los efectos de la interacción competitiva entre las empresas es mediante las reacciones que éstas tienen ante la incertidumbre del mercado, ya sea de costos o demanda (Showalter, 1999, y Jong et al., 2007). Con ello, se decidió incluir en las estimaciones indicadores indirec- 
tos de la incertidumbre, considerando que la incertidumbre del mercado puede reflejarse como la desviación respecto a una tendencia (Showalter, 1999) se definieron tres indicadores indirectos para captar tanto la incertidumbre en la demanda como la incertidumbre en los costos. Para el caso de la incertidumbre en la demanda, los indicadores indirectos propuestos se construyeron con los términos de error obtenidos de tres regresiones de tendencia para el logaritmo de las ventas netas.

$$
\begin{gathered}
\log \left(V_{t}\right)=\beta_{0}+\beta_{1} t+\mathrm{e}_{t} \\
\log \left(V_{t}\right)=\gamma_{0}+\gamma_{1} t+\gamma_{2} t^{2}+v_{t} \\
\log \left(V_{t}\right)=\lambda_{0}+\lambda_{1} t+\lambda_{2} t^{2}+\lambda_{3} t^{3}+v_{t}
\end{gathered}
$$

Algo similar se adoptó para obtener los indicadores indirectos de la incertidumbre en los costos. En este caso, las estimaciones se realizaron con los costos de ventas:

$$
\begin{gathered}
\log \left(C_{t}\right)=\beta_{0}+\beta_{1} t+\mathrm{e}_{t} \\
\log \left(C_{t}\right)=\gamma_{0}+\gamma_{1} t+\gamma_{2} t^{2}+v_{t} \\
\log \left(C_{t}\right)=\lambda_{0}+\lambda_{1} t+\lambda_{2} t^{2}+\lambda_{3} t^{3}+v_{t}
\end{gathered}
$$

Los indicadores indirectos de la incertidumbre en la demanda construidos con (2a) (2b) y (2c) se denotaron en las estimaciones como DEMA, DEMB, DEMC, respectivamente. Mientras que los indicadores indirectos que de la incertidumbre en costos obtenidos con (3a) (3b) y (3c) fueron denotados respectivamente por COSA, COSB y COSC.

En cuanto a los efectos de estas variables sobre la estructura financiera de las empresas, la teoría no es del todo concluyente, y en todo caso menciona que éstos dependerán del entorno competitivo enfrentado por las empresas. Al respecto, si bien Dean Showalter (1995) muestra que bajo un entorno a la Bertrand la incertidumbre en las condiciones de demanda tiene un efecto positivo sobre la deuda y la incertidumbre en costos tiene un efecto negativo, Schuhmacher (2001), por su parte, muestra que los efectos son contrarios a los hallados por Showalter. Mientras, para un entorno a la Cournot la teoría postula que los efectos de la incertidumbre en las condiciones de demanda y en los costos serán positivos. 


\section{RESULTADOS OBTENIDOS}

\section{Clasificación de las empresas}

Para estimar la influencia del entorno competitivo sobre las decisiones financieras de las empresas, es necesario primero clasificarlas de acuerdo con el entorno competitivo enfrentado. Tomando del panel construido para este efecto los datos de las empresas en las cuales se definió alguna relación de competencia, se estimó la medida de interacción competitiva con la metodología propuesta en la sección anterior. Con ello, la estimación de la CSM se realizó utilizando los datos de las 52 empresas incluidas en las 18 relaciones de competencia halladas, siguiendo el criterio del SIC a dos dígitos. De estas 52 empresas, 23 corresponden a empresas con un entorno competitivo caracterizado por complementos estratégicos, otras 15 por sustitutos estratégicos y las 14 restantes no fue posible ubicarlas de acuerdo con algún entorno competitivo, pues el valor de la CSM de éstas se ubicó entre -0.05 y 0.05 .

Respecto de las variables independientes utilizadas en las estimaciones, si bien en algunos casos los diferentes enfoques teóricos postulan los mismos efectos para algunos de los factores determinantes de la estructura financiera de las empresas, para otros factores, como el tamaño o los impuestos, los efectos sugeridos son ambiguos. En el caso de este documento se sugiere que mientras el tamaño de la empresa afecta negativamente la estructura financiera, es decir, mayor tamaño y menor apalancamiento, la tasa impositiva podría tener un efecto positivo, reflejando las posibles ventajas impositivas de mantener un mayor nivel de deuda. El cuadro 3 resume los signos esperados de las variables consideradas en las estimaciones.

Cuadro 3. Factores determinantes y signos esperados

\begin{tabular}{|l|c|l|}
\hline Variable & Indicador indirecto & Signo \\
\hline Tamaño de empresa & SIZE & Negativo \\
\hline Colateral o medida de activos fijos & TANG & Positivo \\
\hline Liquidez & LIQ & Negativo \\
\hline Riesgo & RISK & Negativo \\
\hline Impuestos & TAX & Positivo \\
\hline Rentabilidad & PROF & Negativo \\
\hline
\end{tabular}


Las estadísticas descriptivas de las variables utilizadas se resumen en el cuadro 4. Incluye las estadísticas para el caso de las 91 empresas del panel y para las 52 empresas que mantienen una relación de competencia de acuerdo con el criterio elegido.

Cuadro 4. Estadísticas de las variables utilizadas

(Total de empresas)

\begin{tabular}{|l|c|c|c|c|c|c|}
\cline { 2 - 7 } \multicolumn{1}{c|}{} & Media & Mediana & Máximo & Mínimo & $\begin{array}{c}\text { Desv. } \\
\text { estándar }\end{array}$ & Observaciones \\
\hline LEVB & 0.4908 & 0.4914 & 1.3193 & 0.0294 & 0.1841 & 1983 \\
\hline SIZE & 3.3620 & 3.4058 & 5.2868 & 0.2532 & 0.8318 & 1983 \\
\hline TANG & 0.4762 & 0.5116 & 0.9764 & 0.0031 & 0.2152 & 1983 \\
\hline LIQ & 2.1233 & 1.5697 & 18.8494 & 0.0428 & 1.9135 & 1983 \\
\hline RISK & 0.0152 & 0.0089 & 0.2811 & 0.0000 & 0.0200 & 1983 \\
\hline TAX & -1.5380 & 0.0000 & 330.0191 & -1817.99 & 49.7570 & 1983 \\
\hline PROF & 0.0732 & 0.0732 & 0.3548 & -0.5904 & 0.0756 & 1983 \\
\hline
\end{tabular}

Empresas con relaciones de competencia

\begin{tabular}{|l|c|c|c|r|r|c|}
\cline { 2 - 7 } \multicolumn{1}{c|}{} & Media & Mediana & Máximo & \multicolumn{1}{c|}{ Mínimo } & $\begin{array}{c}\text { Desv. } \\
\text { estándar }\end{array}$ & Observaciones \\
\hline LEVB & 0.5119 & 0.5204 & 1.3124 & 0.0294 & 0.1734 & 1144 \\
\hline SIZE & 3.4043 & 3.5236 & 5.2868 & 0.7342 & 0.8638 & 1144 \\
\hline TANG & 0.4984 & 0.5625 & 0.9764 & 0.0216 & 0.2391 & 1144 \\
\hline LIQ & 1.9166 & 1.4410 & 18.8494 & 0.0428 & 1.8007 & 1144 \\
\hline RISK & 0.0157 & 0.0088 & 0.2811 & 0.0003 & 0.0225 & 1144 \\
\hline TAX & -2.5365 & -0.0553 & 330.0191 & -1817.99 & 65.1922 & 1144 \\
\hline PROF & 0.0664 & 0.0600 & 0.3448 & -0.5904 & 0.0807 & 1144 \\
\hline
\end{tabular}

Por su parte, en el cuadro 5 se muestran las estadísticas descriptivas de las empresas clasificadas de acuerdo con el entorno competitivo enfrentado, es decir, para las empresas que compiten en sustitutos estratégicos (Cournot) y las que compiten en complementos estratégicos (Bertrand).

Revisando las estadísticas del cuadro 5, es posible ver que el tamaño promedio de las empresas en ambos entornos competitivos es similar; el nivel medio de apalancamiento es ligeramente mayor para aquellas empresas que compiten en un entorno a la Bertrand (52\% de los activos totales), mientras que las 
empresas a la Cournot presentan un nivel de $47 \%$ del total de activos. Respecto de la rentabilidad, las empresas que enfrentan una competencia a la Cournot presentan una rentabilidad promedio de $10 \%$, mientras que las empresas con un entorno a la Bertrand sólo alcanzan un promedio de rentabilidad cercano a 5\%. Un dato que resalta es la mayor liquidez promedio de las empresas a la Cournot.

Cuadro 5. Estadísticas de las variables utilizadas por tipo de entorno competitivo (Empresas que compiten en sustitutos estratégicos)

\begin{tabular}{|l|c|r|r|r|r|c|}
\cline { 2 - 7 } \multicolumn{1}{c|}{} & Media & Mediana & Máximo & \multicolumn{1}{c|}{ Mínimo } & $\begin{array}{c}\text { Desv. } \\
\text { estándar }\end{array}$ & Observaciones \\
\hline LEVB & 0.4701 & 0.4788 & 0.8179 & 0.1156 & 0.1633 & 330 \\
\hline SIZE & 3.3265 & 3.3754 & 4.6833 & 1.7500 & 0.6803 & 330 \\
\hline TANG & 0.4913 & 0.6038 & 0.8278 & 0.0470 & 0.2472 & 330 \\
\hline LIQ & 2.6968 & 1.7031 & 18.8494 & 0.3085 & 2.7160 & 330 \\
\hline RISK & 0.0107 & 0.0048 & 0.0833 & 0.0001 & 0.0152 & 330 \\
\hline TAX & -2.2508 & -0.0799 & 236.6047 & -1040.55 & 59.4515 & 330 \\
\hline PROF & 0.1010 & 0.0959 & 0.2601 & -0.1112 & 0.0706 & 330 \\
\hline
\end{tabular}

Empresas que compiten en complementos estratégicos

\begin{tabular}{|l|r|r|r|r|r|c|}
\cline { 2 - 7 } \multicolumn{1}{c|}{} & \multicolumn{1}{c|}{ Media } & Mediana & Máximo & \multicolumn{1}{c|}{ Mínimo } & $\begin{array}{r}\text { Desv. } \\
\text { estándar }\end{array}$ & Observaciones \\
\hline LEVB & 0.5239 & 0.5278 & 1.3124 & 0.0294 & 0.1830 & 506 \\
\hline SIZE & 3.4399 & 3.5715 & 5.2118 & 0.7342 & 0.8486 & 506 \\
\hline TANG & 0.5262 & 0.5708 & 0.9764 & 0.0442 & 0.2047 & 506 \\
\hline LIQ & 1.6523 & 1.2488 & 7.8005 & 0.0851 & 1.2350 & 506 \\
\hline RISK & 0.0153 & 0.0080 & 0.2526 & 0.0002 & 0.0250 & 506 \\
\hline TAX & -3.8881 & -0.0553 & 330.0191 & -1817.99 & 84.4905 & 506 \\
\hline PROF & 0.0482 & 0.0461 & 0.3448 & -0.5904 & 0.0865 & 506 \\
\hline
\end{tabular}

\section{Modelo de efectos fijos}

En la sección anterior se mencionaron las características del modelo de efectos fijos. Para la estimación realizada en este documento, la elección del modelo de efectos fijos se basó en el hecho de que pueden existir efectos individuales no observables correlacionados con los regresores. Se realizaron cuatro ejercicios 
econométricos. El primero consistió en estimar un modelo que sólo considerase los principales factores que determinan la estructura financiera de las empresas. La estimación se realizó con los datos de todas las empresas del panel, por lo que este ejercicio no consideró de modo alguno ni el entorno competitivo ni la interrelación competitiva entre las empresas. Es de mencionar que al estimar las regresiones éstas presentaron problemas de autocorrelación; para corregir estos problemas se incluyó en las especificaciones un AR(1). Así, la especificación de este primer ejercicio está definida por la siguiente ecuación.

$$
\begin{aligned}
\mathrm{LEVB}=c+ & \beta_{1} \mathrm{TANG}+\beta_{2} \mathrm{SIZE}+\beta_{3} \mathrm{RISK}+\beta_{4} \mathrm{PROF} \\
& +\beta_{5} \mathrm{LIQ}+\beta_{6} \mathrm{TAX}+\mathrm{AR}(1)
\end{aligned}
$$

Los resultados del primer ejercicio se resumen en el cuadro 6. Éstos muestran que de los factores determinantes incluidos, la rentabilidad de las empresas (PROF) es uno de los factores más importantes para explicar la estructura financiera de las empresas. En este caso, se sugiere que las empresas preferirán financiarse con flujos financieros internos, que representan fuentes de recursos más baratas respecto de la emisión de deuda. Calculando los efectos, ceteris paribus, por cambios en la rentabilidad promedio de las empresas, se tiene que el efecto de un incremento de poco menos de 4 puntos porcentuales en la rentabilidad promedio, disminuiría el apalancamiento en 1.2 puntos porcentuales. ${ }^{25}$

La importancia de las fuentes internas de recursos de las empresas será corroborada por el indicador indirecto de la liquidez (LIQ), ya que si bien el coeficiente obtenido tiene tanto una alta significación estadística como el signo esperado (efecto negativo sobre la estructura financiera) su nivel es pequeño. Respecto de los demás factores incluidos en este ejercicio, es de notar que tanto el colateral (TANG) como el tamaño (SIZE), si bien son estadísticamente significativos coeficientes no tienen los signos esperados. ${ }^{26}$ Por su parte, el indicador indirecto del riesgo (RISK) y de la tasa impositiva (TAX) no resultaron estadísticamente significativos.

\footnotetext{
${ }^{25}$ La media de la rentabilidad se muestra en el cuadro 4. El aumento en menos de 4 puntos porcentuales de la rentabilidad promedio representa un incremento relativo de $50 \%$ de la misma.

${ }^{26}$ Como se ha mencionado, en el caso de los efectos del tamaño de la empresa sobre la estructura financiera son ambiguos.
} 
Cuadro 6. Resultados del primer ejercicio

Primer Ejercicio

(Total de empresas)

\begin{tabular}{|l|c|c|}
\hline & Coeficiente & $t$-estadístico \\
\hline C & 0.6132 & $34.7902 * * *$ \\
\hline TANG & -0.1910 & $-7.2723 * * *$ \\
\hline SIZE & 0.0053 & $1.7497 *$ \\
\hline RISK & 0.1677 & 1.6179 \\
\hline PROF & -0.3210 & $-7.8831 * * *$ \\
\hline LIQ & -0.0136 & $-9.1048 * * *$ \\
\hline TAX & 0.0000 & 0.1841 \\
\hline AR(1) & 0.8101 & $59.6909 * * *$ \\
\hline$R^{2}$ & 0.9450 & \\
\hline DW & 1.9777 & \\
\hline
\end{tabular}

Significación estadística: $* * * 1 \%, * * 5 \%$ y * $10 \%$.

Como resumen de este primer ejercicio, es posible decir que en el caso de las empresas inscritas en la BMV, se corrobora parcialmente lo propuesto por la teoría respecto a los factores determinantes de la estructura financiera de las empresas, destacando que las empresas son especialmente sensibles al uso de fuentes internas de financiamiento. Un segundo ejercicio econométrico consistió en considerar una especificación que incluyera los diferentes indicadores indirectos de la incertidumbre en la demanda y en los costos. Para este ejercicio se consideraron solamente los datos de las empresas en las cuales fue posible identificar alguna relación competitiva.

Las tres ecuaciones siguientes muestran las especificaciones sugeridas para este ejercicio. En éstas DEMA, DEMB y DEMC, son los indicadores indirectos de la incertidumbre en la demanda, mientras que COSA, COSB y COSC, son los de la incertidumbre en los costos. ${ }^{27}$

$$
\begin{gathered}
\mathrm{LEVB}=c+\beta_{1} \mathrm{TANG}+\beta_{2} \mathrm{SIZE}+\beta_{3} \mathrm{RISK}+\beta_{4} \mathrm{PROF} \\
+\beta_{5} \mathrm{LIQ}+\beta_{6} \mathrm{TAX}+\beta_{7} \mathrm{DEMA}+\beta_{8} \mathrm{COSA}+\mathrm{AR}(1)
\end{gathered}
$$

${ }^{27}$ Estos indicadores indirectos se estimaron con las ecuaciones (2a), (2b), (2c), (3a), (3b) y (3c), explicadas en la sección III del documento. 
34 ECONOMÍA: TEORÍA Y PRÁCTICA • Nueva Época, número 31, julio-diciembre 2009

$$
\begin{aligned}
& \mathrm{LEVB}=c+\beta_{1} \mathrm{TANG}+\beta_{2} \mathrm{SIZE}+\beta_{3} \mathrm{RISK}+\beta_{4} \mathrm{PROF} \\
& +\beta_{5} \mathrm{LIQ}+\beta_{6} \mathrm{TAX}+\beta_{7} \mathrm{DEMB}+\beta_{8} \mathrm{COSB}+\mathrm{AR}(1) \\
& \mathrm{LEVB}=\mathrm{c}+\beta_{1} \mathrm{TANG}+\beta_{2} \mathrm{SIZE}+\beta_{3} \mathrm{RISK}+\beta_{4} \mathrm{PROF} \\
& +\beta_{5} \mathrm{LIQ}+\beta_{6} \mathrm{TAX}+\beta_{7} \mathrm{DEMB}+\beta_{8} \mathrm{COSB}+\mathrm{AR}(1)
\end{aligned}
$$

Los efectos esperados sobre la estructura financiera de las empresas, de los indicadores indirectos en la incertidumbre, en las condiciones de la demanda y en los costos dependerán, como se mencionó, en general del entorno competitivo enfrentado por las empresas. Los resultados del segundo ejercicio se muestran en el cuadro 7, el cual contiene los resultados de las tres diferentes especificaciones, las cuales varían entre sí por la definición considerada de los indicadores indirectos sobre la incertidumbre de la demanda y de los costos.

\section{Cuadro 7. Resultados del segundo ejercicio}

Segundo Ejercicio

(Con relaciones de competencia)

\begin{tabular}{|l|c|c|c|c|c|c|}
\hline & Coeficiente & $t$-estadístico & Coeficiente & $t$-estadístico & Coeficiente & $t$-estadístico \\
\hline C & 0.9937 & $7.8812 * * *$ & 1.1742 & $6.1670 * * *$ & 1.1708 & $7.9449 * * *$ \\
\hline TANG & -0.3175 & $-7.0014 * * *$ & -0.3238 & $-7.0783 * * *$ & -0.3216 & $-7.1146 * * *$ \\
\hline SIZE & -0.0842 & $-2.3975 * *$ & -0.1365 & $-2.5142 * *$ & -0.1374 & $-3.2440 * * *$ \\
\hline RISK & 0.3579 & $2.8259 * * *$ & 0.3546 & $2.7976 * * *$ & 0.3617 & $2.8585 * * *$ \\
\hline PROF & -0.2665 & $-5.0162 * * *$ & -0.2555 & $-4.7945 * * *$ & -0.2520 & $-4.7474 * * *$ \\
\hline LIQ & -0.0142 & $-7.2773 * * *$ & -0.0143 & $-7.3095 * * *$ & -0.0142 & $-7.2911 * * *$ \\
\hline TAX & 0.0000 & 0.1121 & 0.0000 & 0.0984 & 0.0000 & 0.0863 \\
\hline DEMA & -0.0063 & -1.5003 & & & & \\
\hline COSA & 0.0437 & $2.9426 * * *$ & & & & \\
\hline DEMB & & & 0.0271 & 0.9529 & & \\
\hline COSB & & & 0.0335 & $1.9582 * *$ & & \\
\hline DEMC & & & & & 0.0277 & 1.1061 \\
\hline COSC & & & & & 0.0340 & $1.9742 * *$ \\
\hline AR(1) & 0.7963 & $42.0417 * * *$ & 0.7951 & $42.5209 * * *$ & 0.7940 & $42.7007 * * *$ \\
\hline$R^{2}$ & 0.9328 & & $\mathrm{R}^{2}$ & 0.9328 & & 0.9331 \\
\hline DW & 1.9749 & & $\mathrm{DW}$ & 1.9649 & $\mathrm{R}$ & 1.9665 \\
\hline
\end{tabular}

Significación estadística: $* * * 1 \%, * * 5 \%$ y * $10 \%$. 
En este ejercicio, la rentabilidad y la liquidez de las empresas son factores determinantes que explican su estructura financiera. Al respecto, se tiene que para las tres especificaciones un incremento en la rentabilidad promedio de 3.3 puntos porcentuales disminuiría el apalancamiento promedio de las empresas en poco menos de $1 \%(0.93)$.

En cuanto al tamaño de las empresas, tiene el signo esperado y es estadísticamente significativo, corroborando así la preferencia de las empresas por fuentes internas de financiamiento, puesto que empresas más grandes podrían generar mayores flujos de efectivo y utilizarlos para financiarse. Por su parte, en los indicadores indirectos del colateral y el riesgo, si bien son estadísticamente significativos, los signos hallados no son los esperados, mientras que la tasa impositiva no tiene significación estadística.

Referente a los indicadores indirectos de la incertidumbre en las condiciones de los costos y la demanda, el primero guarda una relación positiva con la estructura financiera de las empresas, lo cual podría estar reflejando los incentivos de las empresas de contar con los recursos necesarios para seguir produciendo ante un entorno cambiante. Al respecto, si el indicador de la incertidumbre en costos se incrementase en una desviación estándar, el apalancamiento de las empresas aumentaría en promedio $2 \% .{ }^{28}$ Por su parte, los coeficientes de la incertidumbre en las condiciones de la demanda no resultaron estadísticamente significativos en ninguna especificación.

A diferencia del primer ejercicio, donde no se considera ninguna relación competitiva, es de notar la importancia relativa de incluir en las estimaciones los indicadores indirectos sobre la incertidumbre en los costos. Sin embargo, este ejercicio poco aporta en relación con la interacción entre decisiones financieras y la competencia en los mercados reales, pues como se ha explicado las reacciones de las empresas dependerán del entorno competitivo al que se enfrentan. Es decir, la posible influencia de la competencia en los mercados reales sobre la estructura financiera de las empresas dependerá de si las empresas compiten en complementos o sustitutos estratégicos.

Las especificaciones propuestas en (5a) (5b) y (5c) se utilizaron para el tercer y el cuarto ejercicio. Éstos consideraron los datos de las empresas según su entorno competitivo. Los resultados de las empresas que compiten en comple-

\footnotetext{
${ }^{28}$ Vale la pena recordar que la variable de incertidumbre en los costos capta todos aquellos factores que desvían los costos de la empresa de su tendencia. En este sentido, se incorporan en esta variable los incrementos en la competencia.
} 
mentos estratégicos se muestran en el cuadro 8. En éste, es posible ver la importancia relativa de la rentabilidad de las empresas como factor determinante de su estructura financiera. Así, si la rentabilidad promedio de estas empresas se incrementase en poco más de 2 puntos porcentuales, el apalancamiento promedio disminuiría en alrededor de un punto porcentual $(0.96) .{ }^{29}$ Lo anterior, reforzado por el efecto sugerido de la liquidez de las empresas, la cual cuenta con el signo esperado y es estadísticamente significativa.

\section{Cuadro 8. Resultados del tercer ejercicio}

Tercer Ejercicio

(Empresas que compiten en complementos estratégicos)

\begin{tabular}{|c|c|c|c|c|c|c|}
\hline & Coeficiente & $t$-estadístico & Coeficiente & $t$-estadístico & Coeficiente & $t$-estadístico \\
\hline $\mathrm{C}$ & 0.9361 & $4.6216 * * *$ & 1.1647 & $3.0681 * * *$ & 1.0378 & $3.8168 * * *$ \\
\hline TANG & -0.3096 & $-3.9234 * * *$ & -0.3040 & $-3.8276 * * *$ & -0.3003 & $-3.8073 * * *$ \\
\hline SIZE & -0.0546 & -0.9989 & -0.1226 & -1.1307 & -0.0873 & -1.1133 \\
\hline RISK & 0.6561 & $2.6844 * * *$ & 0.6321 & $2.5673 * * *$ & 0.6206 & $2.5180 * *$ \\
\hline PROF & -0.3862 & $-4.0466 * * *$ & -0.3442 & $-3.5549 * * *$ & -0.3450 & $-3.5907 * * *$ \\
\hline LIQ & -0.0324 & $-4.9016 * * *$ & -0.0321 & $-4.8164 * * *$ & -0.0322 & $-4.8300 * * *$ \\
\hline TAX & 0.0000 & -0.2833 & 0.0000 & -0.2936 & 0.0000 & -0.2911 \\
\hline DEMA & -0.0157 & $-2.3162 * *$ & & & & \\
\hline $\cos A$ & 0.0363 & 1.6052 & & & & \\
\hline DEMB & & & 0.0138 & 0.2694 & & \\
\hline $\cos B$ & & & 0.0385 & 1.5598 & & \\
\hline DEMC & & & & & -0.0033 & -0.0789 \\
\hline $\cos C$ & & & & & 0.0408 & 1.6372 \\
\hline $\mathrm{AR}(1)$ & 0.7875 & $27.2334 * * *$ & 0.7907 & $27.5480 * * *$ & 0.7896 & $27.3679 * * *$ \\
\hline $\mathrm{R}^{2}$ & 0.9084 & & $\mathrm{R}^{2}$ & 0.9074 & $\mathrm{R}^{2}$ & 0.9075 \\
\hline DW & 1.8613 & & DW & 1.8499 & DW & 1.8474 \\
\hline
\end{tabular}

Significación estadística: ***1\%, **5\% y *10\%.

Por otra parte, el colateral y el riesgo no tienen el signo esperado, no obstante su significación estadística, mientras que aun cuando el tamaño cuenta con el signo predicho, no es estadísticamente significativo. En cuanto a los indicadores indirectos de la incertidumbre en las condiciones de la demanda, en dos de las especificaciones muestran un efecto negativo, aunque sólo la primera definición (DEMA) es estadísticamente significativa. En ésta, un incremento de una desviación estándar de la incertidumbre en las condiciones de la demanda origi-

${ }^{29}$ Los dos puntos porcentuales representan un incremento de $50 \%$ de la rentabilidad promedio. 
naría una disminución en el apalancamiento de poco menos de un punto porcentual (0.97).

De los indicadores indirectos de la incertidumbre en costos, si bien los signos hallados coinciden con trabajos previos, las diferentes definiciones consideradas no resultaron estadísticamente significativas. Los efectos sobre la estructura financiera predichos por aquéllos para la incertidumbre en la demanda y en los costos coinciden con lo encontrado por Schuhmacher (2001). Es decir, un efecto negativo de las condiciones de incertidumbre en la demanda y un efecto positivo de las condiciones de incertidumbre en los costos.

En el cuadro 9 se resumen los resultados de las estimaciones para las empresas con un entorno de sustitutos estratégicos. Si bien la rentabilidad de las empresas ayuda a explicar su estructura financiera, el nivel de significación estadística es menor. Sin embargo, un incremento de la rentabilidad de 50\% (5 puntos porcentuales) resulta en una disminución promedio del apalancamiento de un punto porcentual. De igual manera, los niveles del indicador indirecto de la liquidez, no obstante su significación estadística, son menores respecto de los otros ejercicios econométricos. En el caso del tamaño de la empresa, sólo es estadísticamente significativo en dos de las especificaciones. De nueva cuenta, los signos hallados del colateral y el riesgo no coinciden con lo propuesto por la teoría.

\section{Cuadro 9. Resultados del cuarto ejercicio}

Cuarto Ejercicio

(Empresas que compiten en sustitutos estratégicos)

\begin{tabular}{|c|c|c|c|c|c|c|}
\hline & Coeficiente & $t$-estadístico & Coeficiente & $t$-estadístico & Coeficiente & $t$-estadístico \\
\hline C & 0.9225 & $4.6787 * * *$ & 1.2836 & $7.3540 * * *$ & 1.2280 & $7.7075 * * *$ \\
\hline TANG & -0.3219 & $-5.3698 * * *$ & -0.3883 & $-6.3400 * * *$ & -0.3763 & $-6.2703 * * *$ \\
\hline SIZE & -0.0817 & -1.4428 & -0.1804 & $-3.7393 * * *$ & -0.1673 & $-3.7653 * * *$ \\
\hline RISK & 0.8844 & $3.4681 * * *$ & 0.9178 & $3.6662 * * *$ & 0.9675 & $3.8539 * * *$ \\
\hline PROF & -0.1786 & -1.8110 * & -0.1787 & -1.8680 * & -0.1662 & -1.7314 * \\
\hline LIQ & -0.0077 & $-4.4949 * * *$ & -0.0075 & $-4.4251 * * *$ & -0.0074 & $-4.3777 * * *$ \\
\hline TAX & 0.0000 & 0.9939 & 0.0000 & 0.9083 & 0.0000 & 0.8325 \\
\hline DEMA & 0.0017 & 0.3085 & & & & \\
\hline $\cos A$ & 0.0358 & 1.4360 & & & & \\
\hline DEMB & & & 0.1249 & $2.6519 * * *$ & & \\
\hline COSB & & & -0.0443 & -1.0907 & & \\
\hline DEMC & & & & & 0.1110 & $2.4429 * *$ \\
\hline $\cos C$ & & & & & -0.0353 & -0.8692 \\
\hline $\mathrm{AR}(1)$ & 0.7262 & $17.5584 * * *$ & 0.7045 & $17.6148 * * *$ & 0.6998 & $17.6522 * * *$ \\
\hline $\mathrm{R}^{2}$ & 0.9576 & & $\mathrm{R}^{2}$ & 0.9592 & $\mathrm{R}^{2}$ & 0.9592 \\
\hline DW & 2.3160 & & DW & 2.2874 & DW & 2.2778 \\
\hline
\end{tabular}

Significación estadística: *** $1 \%, * \star 5 \%$ y * $10 \%$. 
Los efectos esperados de la incertidumbre en la demanda, que bajo un entorno de sustitutos estratégicos se espera sean positivos, se cumplen para todas las especificaciones; sin embargo, este indicador indirecto sólo es estadísticamente significativo en dos de las especificaciones. Para estas dos especificaciones (DEMB Y DEMC), el incremento de una desviación estándar en estas variables resultaría en un aumento promedio de 6 puntos porcentuales del apalancamiento. Por su parte, ninguno de los indicadores indirectos de la incertidumbre en costos fue estadísticamente significativo, y sólo la primera especificación tiene el signo esperado.

Los resultados de los diferentes ejercicios realizados muestran que la estructura financiera de las empresas se explica, en parte, por el uso de fuentes de financiamiento internas, como los rendimientos, la liquidez o los flujos generados por el tamaño de empresa. Al respecto, es posible que los ahorros generados por el uso más eficiente de los recursos, producto en parte de economías de escala de la producción debidas al tamaño de la empresa, se traduzcan en mayores flujos de recursos propios para financiarse. Lo anterior, no obstante el signo hallado en el indicador del colateral.

Cabe recordar que en este documento se trató captar el vínculo entre las decisiones financieras y las decisiones de mercado de las empresas, por un lado con la clasificación de las empresas según su entorno competitivo -sustitutos o complementos estratégicos-, y por otro mediante su reacción ante la incertidumbre en las condiciones de mercado, la demanda y los costos. ${ }^{30}$ Con ello, los resultados de los dos últimos ejercicios econométricos muestran la diferencia en la respuesta de las empresas ante un entorno competitivo diferente. Ejemplo de ello es la respuesta hallada ante la incertidumbre en las condiciones de la demanda, en donde, para las empresas que compiten en sustitutos estratégicos (Cournot), el efecto encontrado sobre el nivel de endeudamiento de las empresas es positivo, caso contrario para las empresas con un entorno de complementos estratégicos (Bertrand). Como se mencionó, este hecho coincide con lo hayado por Schuhmacher (2001).

Posiblemente la respuesta diferenciada de las empresas se explique por la mayor sensibilidad a los movimientos en los acervos de las empresas que compiten en sustitutos estratégicos. Es decir, a diferencia de las empresas que compi-

\footnotetext{
${ }^{30}$ La interacción se refleja en las reacciones de las empresas ante cambios de estrategias competitivas de sus rivales y ante modificaciones en la propia estrategia por cambios en la incertidumbre de las condiciones del mercado.
} 
ten en complementos estratégicos, las empresas con un entorno de sustitutos estratégicos, dado que les resulta más difícil el ajuste en su capacidad de producción, pueden tener mayores pérdidas ante cambios repentinos en sus acervos, con ello los incentivos de contratación de deuda son positivos. Así, ante un incremento de la incertidumbre en las condiciones de la demanda, reflejado por desviaciones respecto de la tendencia de las ventas netas, las empresas con un entorno de sustitutos estratégicos podrían incrementar su nivel de endeudamiento con el fin o de permanecer en el mercado ante caídas en las ventas, o de ampliar su capacidad de producción y probablemente orillar la salida de sus competidores ante un aumento en las ventas. Lo anterior quizá sea menos probable para el caso de las empresas que compiten en complementos estratégicos, pues éstas cuentan con mayor flexibilidad en la modificación de su capacidad de producción.

Por otra parte, no obstante los resultados obtenidos, una posible limitación del documento es la omisión de variables que permitan controlar de manera explícita los efectos del entorno macroeconómico, los cuales pueden incidir tanto sobre las decisiones financieras como sobre las decisiones competitivas de las empresas. Entre estas variables se pueden mencionar aquellas asociadas con las restricciones en el acceso al financiamiento o los posibles riesgos cambiarios.

\section{CONCLUSIONES}

Motivado por la importancia que tiene el análisis de las decisiones de las empresas en los diversos mercados donde interactúan, el presente documento aportó evidencia empírica sobre la influencia de las decisiones competitivas de las empresas en sus decisiones financieras, ayudando con ello a cubrir la falta de trabajos empíricos acerca de esta interacción. La evidencia aportada se enmarcó en una economía emergente, como la de México, donde el grado de profundidad e interacción entre los mercados puede ser aún débil.

Los resultados hallados en los diferentes ejercicios realizados corroboran parcialmente la teoría sobre los principales factores determinantes de la estructura financiera de las empresas, pues mientras factores como la tasa impositiva resultaron ser estadísticamente no significativos, otros, como la liquidez o la rentabilidad, obtuvieron el signo esperado y resultaron ser estadísticamente significativos. Destacan estos últimos en el sentido de que revelan la posible preferencia de las empresas por el uso de fuentes internas de recursos como medio de financiamiento, pues son más baratos que la contratación de deuda. 
Por su parte, los resultados hallados al identificar el entorno competitivo y clasificar a las empresas de acuerdo a éste -complementos o sustitutos estratégicos-, así como al estimar la influencia de las decisiones de mercado sobre las decisiones financieras, aportan indicios sobre los efectos diferenciados que tiene el entorno competitivo en la estructura financiera de las empresas. Como se vio, en los resultados para las empresas con un entorno de complementos estratégicos (competencia a la Bertrand), se corrobora lo mencionado por trabajos previos. Además, estos resultados se diferencian de los hallados para empresas que compiten en sustitutos estratégicos (competencia a la Cournot).

Es de resaltar que el presente documento es el primer trabajo que aporta evidencia empírica respecto del vínculo entre decisiones financieras y competitivas de las empresas en México. En este sentido, constituye un intento por cubrir la falta de trabajos empíricos sobre el tema. Sin embargo, también es de reconocer que una posible limitación del análisis realizado es no considerar las estimaciones variables que controlen algunos efectos relacionados con el entorno macroeconómico, los cuales pueden influir en el comportamiento de las empresas. Con información disponible de más mercados sería posible precisar más los resultados obtenidos.

Finalmente, es importante mencionar la vigencia que tiene el tema tratado, pues conocer qué factores determinan la estructura financiera de las empresas y analizar cómo inciden en ésta, resulta importante para comprender mejor las posibles reacciones de las empresas ante modificaciones en las condiciones de los mercados, lo cual podría ayudar en la propuesta de medidas de política que fomenten el desarrollo eficiente de las empresas. 


\section{REFERENCIAS BIBLIOGRÁFICAS}

Bolsa Mexicana de Valores (2007), Informe Anual Bolsa Mexicana de Valores 2007.

Bolton, Patrick, y David Scharfstein (1990), "A theory of predation base on agency problems in financial contracting", American Economic Review, 80 (1), páginas 93-106.

Booth, Laurence, et al. (2001), "Capital structures in developing countries", Journal of Finance, LVI (1), pp. 87-130.

Brander, James, y Tracy Lewis (1986), "Oligopoly and financial structure: The limited liability effect", American Economic Review, 76 (5), pp. 956-970.

Bulow, Jeremy, et al. (1985), "Multimarket oligopoly: strategic substitutes and complements", Journal of Political Economy, 93 (3), pp. 488-511.

Chevalier, Judith (1995), "Capital structure and product-market competition: Empirical evidence from supermarket industry", American Economic Review, 85 (3), pp. 205-256.

_ , y David Scharfstein (1995), "Capital-market imperfections and countercyclical markups: Theory and evidence", American Economic Review, 86 (4), pp. 703-725.

Franck, Tom, y Nancy Huyghebaert (2004), "On the interactions between capital structure and product markets: A survey of the literature", Tijdschrift voor Economie en Management, XLIX (4), pp. 727-787.

Greene, William H. (2000), Análisis econométrico, Prentice Hall, 3ra. ed.

Harris, Milton, y Artur Raviv (1991), "The theory of capital structure", Journal of Finance, XLVI (1), pp. 297-355.

Jong, Abe de, et al. (2007), "Strategic debt: Evidence from Bertrand and Cournot competition", Report Series Research in Management ERS-2007-057-F\&A, Erasmus Research Institute of Management, Erasmus University.

Jorgensen, Jan J., y Paulo R. S. Terra (2003), "Determinants of capital structure in Latin America: The role of firm-specific and macroeconomic factors", McGill University, Documento de Trabajo.

Lyandres, Evgeny (2006), "Capital structure and interaction among firms in output markets: Theory and evidence", Journal of Business, 79 (5), pp. 2381-421.

Mackay, Peter, y Gordon M. Phillips (2005), "How does industry affect firm financial structure?", Review of Financial Studies, 18 (4), pp. 1433-1466.

Maksimovic, Vojislav (1988), "Capital structure in repeated oligopolies", RAND Journal of Economics, 19 (3), pp. 389-407. 
Modigliani, Franco, y Merton H. Miller (1958), "The Cost of Capital, Corporation Finance and the Theory of Investment", American Economic Review, 48 (3), pp. 261-297.

Myers, Stewart C. (2001), “Capital structure”, Journal of Financial Perspectives, 15 (2), pp. 81-102.

Phillips, Gordon (1995), "Increased debt and industry product markets: An empirical analysis", Journal of Financial Economics, 37 (2), pp. 189-238.

Presidencia de la República (2008), Anexo Estadístico del Segundo Informe de Gobierno, México.

Rodríguez Matilla, Max Alberto (2002), Estructura de capital: Una estimación para México, Instituto Tecnológico Autónomo de Monterrey, tesis de maestría en Finanzas.

Rubach Catano, Karl Peter (2004), Estructura de capital óptimo: El caso de México, Instituto Tecnológico Autónomo de Monterrey, tesis de maestría en Finanzas.

Schuhmacher, Frank (2001), "Capacity-Price-Competition and Financial Structure”, Social Science Research Network Working Paper Series, disponible en: http://ssrn. com/abstract $=302407$.

Showalter, Dean (1995), "Oligopoly and financial structure: comment”, American Economic Review, 85 (3), pp. 647-653.

- (1999), "Strategic debt: evidence in manufacturing”, International Journal of Industrial Organization, 17 (3), pp. 319-333.

Stiglitz, Joseph E. (1988), "Why financial structure matters", Journal of Economic Perspectives, 2 (4), pp. 121-126.

Sundaram, et al. (1996), "An empirical analysis of strategic competition and firm values: The case of R\&D competition”, Journal of Financial Economics, 40 (3), pp. 459-486.

Tirole, Jean (2006), The Theory of Corporate Finance, Princeton University Press.

Wooldridge, Jeffrey M. (2002), Econometric Analysis of Cross Section and Panel Data, The MIT Press.

Zingales, Luigi (1998), "Survival of the fittest or the fattest? Exit and financing in the trucking industry”, Journal of Finance, LIII (3), pp. 905-938. 